\title{
High-resolution Kendrick Mass Defect Analysis of Poly(ethylene oxide)-based Non-ionic Surfactants and Their Degradation Products
}

\author{
Thierry Fouquet ${ }^{1, *}$, Haruo Shimada ${ }^{2}$, Katsuyuki Maeno ${ }^{2}$, Kanako Ito ${ }^{3}$, Yuka Ozeki ${ }^{3}$, \\ Shinya Kitagawa ${ }^{3}$, Hajime Ohtani ${ }^{3}$ and Hiroaki Sato ${ }^{1, *}$ \\ ${ }_{1}^{1}$ National Institute of Advanced Industrial Science and Technology (AIST), Research Institute for Sustainable Chemistry, Tsukuba, JAPAN
${ }^{2}$ Shiseido Global Innovation Center, Yokohama, JAPAN \\ ${ }^{3}$ Nagoya Institute of Technology, Graduate School of Engineering, Life Science and Applied Chemistry, Nagoya, JAPAN
}

\begin{abstract}
Matrix assisted laser desorption ionization (MALDI) high-resolution mass spectrometry (HRMS) and the recently introduced high-resolution Kendrick mass defect (HRKMD) analysis are combined to thoroughly characterize non-ionic surfactants made of a poly(ethylene oxide) (PEO) core capped by esters of fatty acids. A PEO monostearate surfactant is first analyzed as a proof of principle of the HRKMD analysis conducted with a fraction of $\mathrm{EO}$ as the base unit (EO/X with $\mathrm{X}$ being an integer) in lieu of EO for a regular KMD analysis. Data visualization is greatly enhanced and the distributions detected in the MALDI mass spectrum are assigned to a pristine $(\mathrm{H}, \mathrm{OH})-\mathrm{PEO}$ as well as mono- and di-esterified PEO chains with palmitate and stearate end-groups in HRKMD plots computed with EO/45. The MALDI-HRMS/HRKMD analysis is then successfully applied to the more complex case of ethoxylated hydrogenated castor oil (EHCO) found to contain a large number of hydrogenated ricinoleate moieties (up to 14) in its HRKMD plot computed with EO/43, departing from the expected triglyceride structure. The exhaustiveness of the MALDI-HRMS/HRKMD strategy is validated by comparing the so-obtained fingerprints with results from alternative techniques (electrospray ionization MS, size exclusion and liquid adsorption chromatography, ion mobility spectrometry). Finally, aged non-ionic surfactants formed upon hydrolytic degradation are analyzed by MALDI-HRMS/HRKMD to easily assign the degradation products and infer the associated degradation routes. In addition to the hydrolysis of the ester groups observed for EHCO, chain scissions and new polar end-groups are observed in the HRKMD plot of PEO monostearate arising from a competitive oxidative ageing.
\end{abstract}

Key words: Kendrick mass defect, high-resolution mass spectrometry, poly(ethylene oxide), non-ionic surfactant, hydrolytic degradation, castor oil, stearate

\section{INTRODUCTION}

Surfactants are a class of molecules of prime importance in industry considering their numerous applications as detergents, foaming agents or emulsifiers in cosmetics, cleaning, textile or agrochemical formulations to mention but a few $^{1-6)}$. Oil-soluble surfactants share a common molecular architecture composed of a polar head (hydrophilic) and a nonpolar tail (hydrophobic / lipophilic, typically a long hydrocarbon chain C12-C20) and are conveniently classified according to the nature of the polar head ${ }^{7)}$. Four main classes are usually considered and known as anionic surfactants (negatively charged polar head group), cationic

\begin{abstract}
Abbreviations: CNKM, corrected nominal Kendrick mass; CO, castor oil; EHCO, ethoxylated hydrogenated castor oil; EO, ethylene oxide; ESI, electrospray ionization; HCO, hydrogenated castor oil; HR, high-resolution; IM, ion mobility; KM, Kendrick mass; KMD, Kendrick mass defect; LAC, liquid adsorption chromatography; LCCC, liquid chromatography at the critical condition; MALDI, matrix assisted laser desorption ionization; MS, mass spectrometry; $\mathrm{MS}^{\mathrm{n}}$, multistage mass spectrometry; MS/MS, tandem mass spectrometry; NKM, nominal Kendrick mass; PEO, poly (ethylene oxide); RID, refractive index detector; SEC, size exclusion chromatography.
\end{abstract}

\footnotetext{
* Correspondence to: Thierry Fouquet, National Institute of Advanced Industrial Science and Technology (AIST), Research Institute for Sustainable Chemistry, Tsukuba, JAPAN

E-mail: thierry.fouquet@aist.go.jp. Hiroaki Sato, e-mail :sato-hiroaki@ aist.go.jp

Accepted April 20, 2017 (received for review April 9, 2017)

Journal of Oleo Science ISSN 1345-8957 print / ISSN 1347-3352 online

http://www.jstage.jst.go.jp/browse/jos/ http://mc.manusriptcentral.com/jjocs
} 
surfactants (positively charged polar head group), amphoteric surfactants (both positive and negative charges borne by the polar head group) and non-ionic surfactants (neutral polar head group). The latter have increased their market share in the last decades and are now a non-negligible part of the total surfactant production worldwide ${ }^{1)}$. Non-ionic surfactants are found in a large variety of pharmaceuticals and cosmetics thanks to their low toxicity and good compatibility with other components of complex formulations.

Among the most widely used non-ionic surfactants, ethoxylated chemicals formed upon the polycondensation of ethylene oxide on a polar group (e.g. alcohol, amine) are prominent and referred to as poly (ethylene oxide)-like surfactants (PEO-like, the PEO chain acting as the polar head). A typical example is the so-called ethoxylated (hydrogenated or pristine) castor oil ${ }^{8,9)}$. Castor oil (CO) is a valuable seed oil extracted from the seeds of Ricinus communis (vernacular name: castor oil plant) and usually described as a triglyceride of ricinoleic acid(i.e. ester of glycerol and ricinoleic acid, $\mathrm{C}_{18} \mathrm{H}_{34} \mathrm{O}_{3}, 298 \mathrm{Da}$, also known as 12-hydroxystearic acid). Several applications have been reported for $\mathrm{CO}$ such as the formulation of polyurethanes ${ }^{10-16)}$ similarly to its bio-based congeners extracted from plants (e.g. cardanol). The hydroxyl group $-\mathrm{OH}$ carried by the $12^{\text {th }}$ carbon and the unsaturation between the $9^{\text {th }}$ and $10^{\text {th }}$ carbon atoms of the $\mathrm{C} 18$ chain makes ricinoleic acid unique among fatty acids as a tremendous platform for additional chemical functionalizations. The hydrogenated $\mathrm{CO}(\mathrm{HCO}$, also known as castor wax) is produced by the hydrogenation of the ricinoleate moieties (to produce a hydrogenated ricinoleic acid, $\mathrm{C}_{18} \mathrm{H}_{36} \mathrm{O}_{3}, 300 \mathrm{Da}$ ) and typically used in cosmetics and lubricants. The ethoxylation of HCO finally leads to a so-called ethoxylated hydrogenated castor oil (noted EHCO, also known as hydrogenated castor oil ethoxylate, poly (ethylene glycol)hydrogenated castor oil, polyoxoethylene hardened castor oil, polyoxoethylene hydrogenated castor oil or castor wax ethoxylate) used as an emuslifier, solubilizer or lubricant ${ }^{17)}$. $\mathrm{CO}, \mathrm{HCO}$ and ethoxylated castor oil (ECO, no hydrogenation of the unsaturations) have been extensively described using a great variety of analytical techniques ${ }^{18-24)}$ while EHCO has not been extensively characterized so far.

Relying on the capabilities the matrix assisted laser desorption ionization (MALDI) high-resolution (HR) mass spectrometry (MS) analysis for the thorough analysis of polymers ${ }^{25)}$, the present article deals with the characterization of a EHCO sample by combining a MALDI-HRMS step with a newly introduced high-resolution Kendrick mass defect analysis (HRKMD). A HRKMD analysis is based on the use of a fraction of the repeat unit of a polymeric backbone (repeat unit) $/ X$ with $X$ being a positive integer ${ }^{26-28}$ instead of the repeat unit itself as traditionally done for the regular KMD analysis of polymer ions ${ }^{29-32)}$. Such a fractional base unit has been found to greatly amplify the variations of
$\mathrm{KMD}$ (e.g. between isotopes from the isotope distribution of a given oligomer or between components of a blend), producing a so-called "high-resolution" KMD plot and constituting a breakthrough in the data mining of mass spectral data using the KMD analysis. Its combination with a direct MALDI-HRMS analysis is proposed here for a rapid but complete screening of complex samples such as EHCO. Being implemented for the characterization of surfactants for the first time, a "simpler" PEO monostearate sample ${ }^{33-35)}$ (also known as poly(ethylene glycol) stearate, PEG stearate, polyoxoethylene stearate) is used as a reference sample to check at the capability of the strategy prior to its application for a notoriously complex EHCO.

The representativeness of a direct MALDI-HRMS / HRKMD analysis is also validated by comparing the so-obtained data with alternative ion sources (namely electrospray) and fractionation of the sample by size exclusion chromatography (SEC), liquid adsorption chromatography $(\mathrm{LAC})^{36}$, liquid chromatography at the critical condition $(\text { LCCC })^{37-39)}$ and ion mobility (IM) ${ }^{40)}$. Exemplifying its sofound high capabilities, MALDI-HRMS / HRKMD is finally extended for the rapid analysis of the aged PEO monostearate and EHCO after their hydrolytic degradation, the stability of surfactants and their degradation products being of top importance for the formulation of safe and ecofriendly finished products for every application. The research plan is summed up in Fig. 1 which also provides very schematic representations of the analytical techniques used in this study to highlight their principles.

\section{MATERIALS AND METHODS}

\subsection{Chemicals and degradation procedure}

An extended "Materials and Methods" section is available in the Supporting Information for experimental details about ESI-MS and multistage MS (ESI-MS $\left.{ }^{\mathrm{n}}\right)$, LAC-MS, LCCC-MS and IM-MS.

Trans-2-[3-(4-tert-butylphenyl)-2-methyl-2-propenylidene]-malononitrile (known as DCTB) was from Tokyo Chemical Industry Co., Ltd(TCI, Tokyo, Japan). PEO "monostearate", EHCO 10 EO (further noted EHCO for the sake of simplicity) and chloroform $\left(\mathrm{CHCl}_{3}\right)$ were purchased from Wako Pure Chemical Industries (Osaka, Japan). Chemicals were used as received without purification.

For the degradation tests, stable emulsions of PEO "monostearate" and EHCO at $1 \mathrm{mg} \mathrm{mL}^{-1}$ were prepared in phosphate buffers at $\mathrm{pH} 7.00$ and let to react at $40^{\circ} \mathrm{C}$ using a thermostated-controlled device in static mode (no agitation). Samples were recovered after 15 days of ageing.

\subsection{Size exclusion chromatography}

Size exclusion chromatography (SEC) fractionations were performed using a HLC8220 GPC system(Tosoh, 


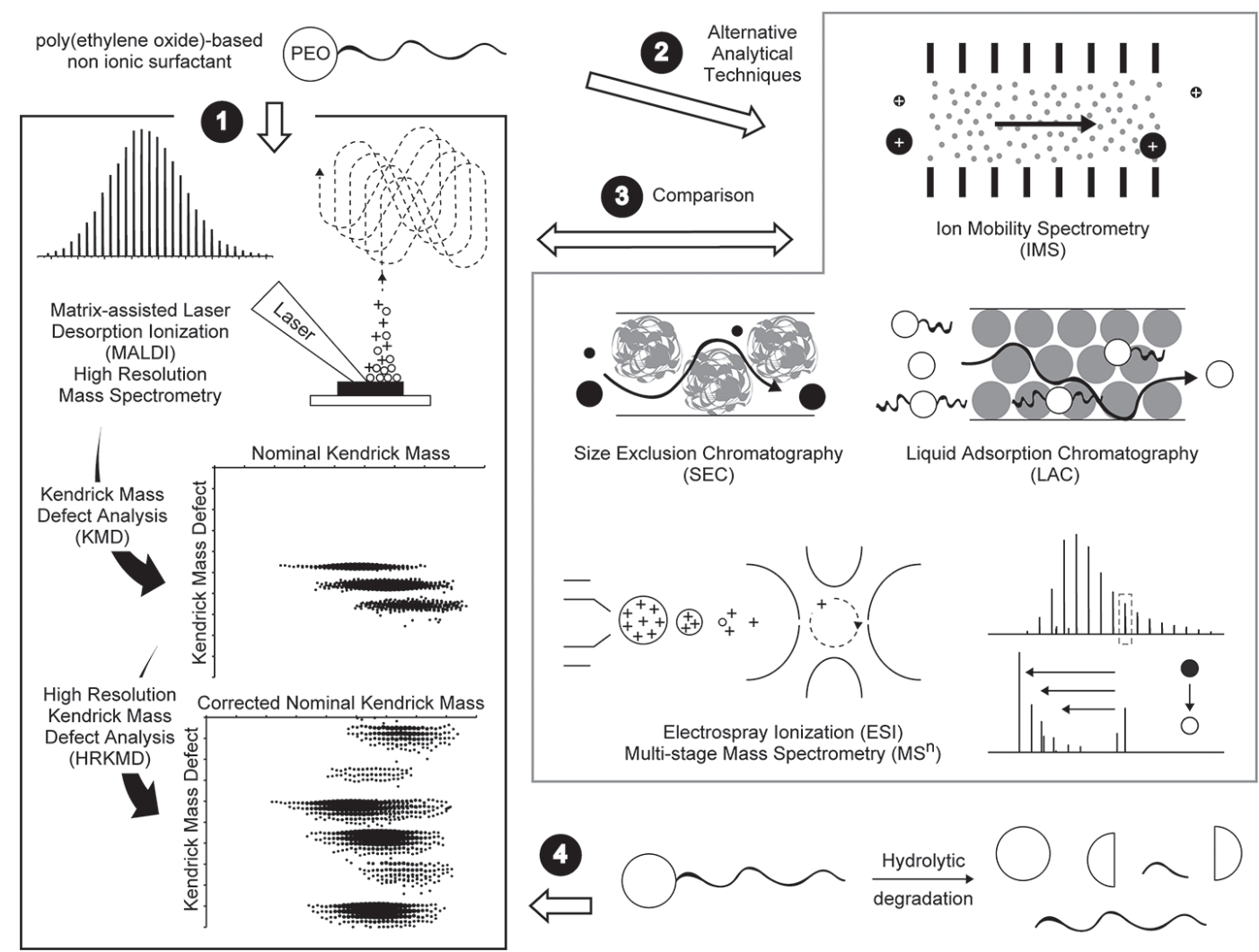

Fig. 1 Analytical strategy proposed to comprehensively characterize PEO-based non-ionic surfactants. (1) Combination of MALDI-HRMS and HRKMD. (2) Alternative techniques (IMS, SEC, LAC and ESI-MS ${ }^{\mathrm{n}}$ ) to check at the representativeness of MALDI-HRMS / HRKMD (3). (4) Application of MALDI-HRMS and HRKMD to hydrolyzed surfactants.

Tokyo, Japan) equipped with a refractive index detector (RID). Separation was carried out using two TSKgel multipore $\mathrm{H}_{\mathrm{XL}}-\mathrm{M}$ columns $(7.8 \mathrm{~mm} \times 300 \mathrm{~mm})$ connected in series following a multipore Hxl guard column. $\mathrm{CHCl}_{3}$ was used as the mobile phase at a $1 \mathrm{~mL} \mathrm{~min}^{-1}$ flow rate. Surfactants were dissolved in $\mathrm{CHCl}_{3}$ at $2 \mathrm{mg} \mathrm{mL}^{-1}$, filtered using Millex syringe-driven filter units (Merck Millipore, Carrigtwohill, Ireland) and $200 \mu \mathrm{L}$ of the so-formed solution were injected for the SEC elutions. The fractionation was done by collecting aliquots of $0.5 \mathrm{~mL}$ (30 seconds) into vials directly after the RID further allowed to air dry for a couple of hours. Following this re-concentration procedure, the sensitivity of the mass spectrometer allowed satisfactory mass spectra to be recorded from one single analytical SEC run only.

\subsection{Mass spectrometry}

The MALDI mass spectra of the pristine and aged surfactants were recorded using a JMS-S3000 SpiralTOF mass spectrometer $^{41)}$ (JEOL, Tokyo, Japan) equipped with a Neodymium-doped yttrium lithium fluoride laser irradiating the deposits $\left(1 \mu \mathrm{L}\right.$ of a solution of analyte at $1 \mathrm{mg} \mathrm{mL}^{-1}$ and DCTB at $15 \mathrm{mg} \mathrm{mL}^{-1} 50 / 50 \mathrm{vol} \%$ deposited on a 384 circle surface from Hudson Surface Technology, Old Trappan,
$\mathrm{NJ})$. The delay time was set at $350 \mathrm{~ns}$ to keep the peak width $\Delta \mathrm{M}<0.03 \mathrm{Da}$ at FWHM over the mass range of interest. Calibration was performed externally and internally using the sodium adducts of a poly (methyl methacrylate) $1310 \mathrm{~g} \mathrm{~mol}^{-1}$ and a hydroxyl-ended polydimethylsiloxane $550 \mathrm{~g} \mathrm{~mol}^{-1}$ standards (DCTB, no salt added). MSTornado control/analysis (JEOL) was used for data recording and preliminary data treatment while mMass 5.5.0.0 was used for data treatment and artworks ${ }^{42)}$. Data points have been peak-picked from the mass spectra using an automated procedure implemented in mMass with no deisotoping and a relative intensity threshold set at $0.5 \%$.

\subsection{Kendrick mass defect analysis}

The measured mass-to-charge ratios $(\mathrm{m} / \mathrm{z})$ were converted to Kendrick masses (KMs), nominal Kendrick masses (NKMs) and Kendrick masses defects (KMDs) using a fraction of the ethylene oxide repeat unit as the base unit according to:

$$
\mathrm{KM}=m / z \cdot \frac{44 / X}{44.0262 / X}
$$

$\mathrm{NKM}=\operatorname{round}(\mathrm{KM})$ 
$\mathrm{KMD}=\mathrm{NKM}-\mathrm{KM}$

with "round()" being the nearest integer function and $\mathrm{X}$ being a positive integer $\left(\mathrm{X}=1\right.$ for a regular KMD analysis $^{29-32)}$, $\mathrm{X}=43,45$ and 87 for a HRKMD analysis ${ }^{26-28)}$. . A corrected nominal Kendrick mass value (CNKM) was finally considered to prevent any shift of the KMD plot and calculated as follows:

$$
\mathrm{CNKM}=\mathrm{NKM}-\operatorname{ceiling}(\mathrm{NKM}-m / z)
$$

with "ceiling $(\mathrm{x})$ " the smallest integer greater than or equal to $\mathrm{x}$. A KMD plot displays the KMD of the oligomeric adducts as a function of their CNKM using a "bubble chart" where each disk expresses a data triplet (CNKM, KMD, abundance ${ }^{26-32)}$. "Full scale" will be used throughout the text to designate a KMD plot with KMD ranging from -0.5 to 0.5 , its minimum and maximum values by definition.

\section{RESULTS AND DISCUSSION}

\subsection{MALDI-HRMS / HRKMD analysis of PEO "monostea-} rate"

A relatively simple PEO "monostearate" sample has been characterized first as a proof of principle of the methodology combining a MALDI-HRMS and a HRKMD analyses. A representative MALDI mass spectrum is depicted in Fig. 2A and displays a set of PEO distributions (consecutive oligomers spaced by 44.0262 Da, i.e. EO unit) centered around $2000 \mathrm{Da}$ in accordance with the "40 EO" content mentioned by the supplier. Looking closer (insets in Fig. 2A left), six main distributions are detected thanks to the high resolving power of the SpiralTOF mass analyzer ${ }^{41}$. Rather than a time consuming exploration of the mass spectrum through a manual assignment peak by peak but relying on the simulation of KMD readily computed from a generic composition, assignments of ion series is done far more easily using a KMD plot. In particular, since the distribution in terms of EO units is turned into a single horizontal line in a EO-based KMD plot, simulating one KMD value is enough to assign all the peaks of a given $\mathrm{PEO}$ ion series.

A regular KMD plot computed with EO as the base unit is depicted in Fig. 2B and displays three main clouds of points lining up horizontally. A first line at KMD $\sim 0.02$ in the low NKM range is assigned to a $(\mathrm{H}, \mathrm{OH})$-ended PEO series (i.e. unreacted poly (ethylene) glycol) and noted (1), based on the KMD value and further confirmed by the accurate mass measurements (Table S1, Supporting Information). The expected monostearate ( $\mathrm{H}$, stearate)-PEO series is detected in the medium NKM range at KMD $\sim-0.07$ (noted 2a), supposedly formed upon the mono-esterification of a $(\mathrm{H}, \mathrm{OH})-\mathrm{PEO}$ chain by one stearic acid moiety (octadecanoic acid $\mathrm{C} 18$, inset in Fig. 2A right). If $(\mathrm{H}, \mathrm{OH})-\mathrm{PEO}$ is the residual reagent for a monocapping reaction, a (stea-

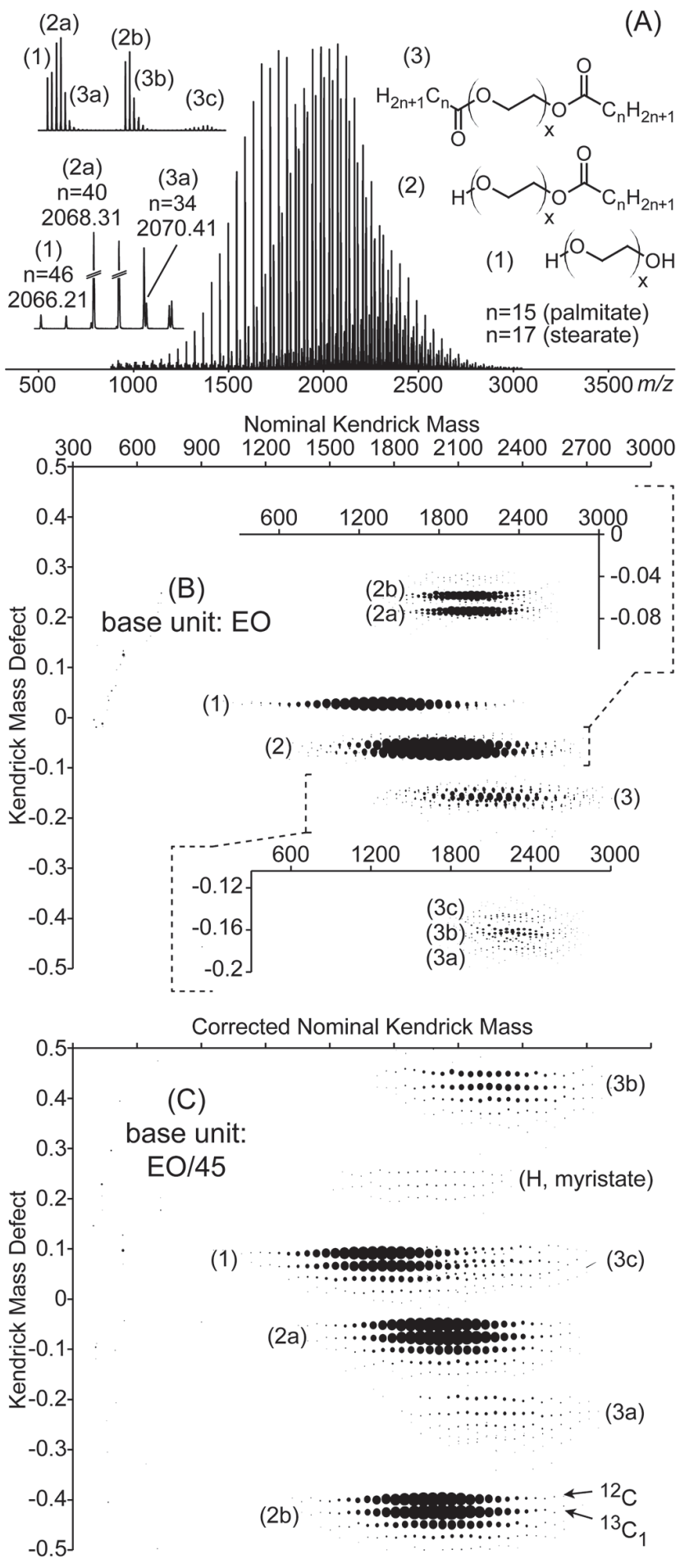

Fig. 2 (A) MALDI mass spectrum of PEO "monostearate". Insets: magnifications of the mass spectrum and structures of the main components $((1):(\mathrm{H}, \mathrm{OH})$, (2a): (H, stearate), (2b): (H, palmitate), (3a): (stearate, stearate), (3b): (palmitate, stearate), (3c): (palmitate, palmitate)-ended PEOs). (B) Regular KMD plot computed with EO. (C) HRKMD plot computed with EO/45. 
rate, stearate)-PEO by-product formed upon its di-esterification is also observed in the high NKM range at KMD -0.17 and noted $3 \mathrm{a}$. Back to the mass spectrum, the need for a high-resolution mass analyzer is exemplified with the differentiation between $(2 \mathrm{a})$ and $(3 \mathrm{a}):{ }^{13} \mathrm{C}_{2}$ of $(2 \mathrm{a}, \mathrm{n}=40)$ at $\mathrm{m} / \approx 2070.3160$ differs from ${ }^{12} \mathrm{C}$ of $(3 \mathrm{a}, \mathrm{n}=34)$ at $\mathrm{m} / \mathrm{z}$ 2070.4130 of $\Delta \mathrm{m}=97 \mathrm{mDa}(\delta=47 \mathrm{ppm}$, inset in Fig. $2 \mathrm{~A}$ left). Distinguishing the degree of esterification is thus done at first sight in the KMD plot at full scale but a magnification of the KMD plot (insets in Fig. 2B) is required to reveal three other distributions assigned to a mono-esterified ( $\mathrm{H}$, palmitate)-PEO and di-esterified (palmitate, stearate) $-\mathrm{PEO}$ and (palmitate, palmitate)-PEO noted $2 \mathrm{~b}, 3 \mathrm{~b}$ and $3 c$, respectively (limited variation of KMD: (2a) vs. (2b), $\Delta \mathrm{KMD} \sim+0.0146)$. In addition to $(\mathrm{H}, \mathrm{OH})$-PEO and stearic acid, palmitic acid (hexadecanoic acid C16, inset in Fig. $2 \mathrm{~A}$ right) has thus been used in the preparation of this non-ionic surfactant as an unexpected secondary reagent.

The nature of the fatty acids (stearic acid C18 and palmitic acid C16) and the mono-esterification have been validated by ESI-MS ${ }^{2}$ and MS $^{3}$, respectively (Fig. S1). However, the regular KMD plot is not optimal owing to 1) the absence of isotopic resolution and b) a poor distinction of the three di-esterified series $(3 \mathrm{a}-\mathrm{c})$. The finite resolution / mass accuracy of the MS analysis and the intrinsically low variations of the KMD values accounts for the fuzzy appearance and a limited resolving power of the KMD plot, respectively. To overcome this pitfall, the concept of a fractional base unit has been introduced very recently. Instead of using a molecule or part of a molecule as the base unit for the calculation of Kendrick masses (i.e. base transformation from the IUPAC mass scale to a new Kendrick mass scale) such as $-\mathrm{CH}_{2}$ - in the original method proposed by Kendrick ${ }^{43)}$ or a repeat unit of a polymeric backbone in its modified form $^{29)}$, a fraction of the repeat unit (repeat unit) $X$ with $X$ being an integer is proposed as a chemically senseless but mathematically acceptable base $u_{n i t}{ }^{26)}$. This mathematical trick dramatically amplifies the variation of KMD values and improves the resolution of the KMD plots without interfering with the basics of a KMD analysis such as the point alignments of homologue ions (horizontal or oblique). This new "high-resolution" KMD analysis is readily done manually using a regular spreadsheet software (cf. Experimental Section) or automatically using the associated option now commercially available in dedicated softwares (Mass Mountaineer, RBC software, Portsmouth, NH). The HRKMD plot computed with a fractional $\mathrm{EO} / 45$ as the base unit (Fig. 2C) displays 6 main clouds of points readily assigned at full scale with no need for magnification. With the expansion of the KMD dimension using a fractional base unit, the variation of KMD between a $(\mathrm{H}$, stearate) $-\mathrm{PEO}$ oligomer $(2 \mathrm{a})$ and a ( $\mathrm{H}$, palmitate)-PEO oligomer $(2 \mathrm{~b})$ is now $\sim-0.3487$ (gain of resolution: $\mathrm{x} 24$ ). Thanks to the improvement of resolution, an additional distribution is also barely seen between (1) and (3b) and assigned to a monoesterified ( $\mathrm{H}$, myristate)-PEO series (tetradecanoic acid C14, known as myristic acid). Such a side-product is detected in the MALDI mass spectrum with very low intensity $(<1 \%$, Table S1) and is more easily visualized in a KMD plot. Finally, the HRKMD plot is isotopically resolved at full scale allowing a selection of monoisotopic peaks only in a so-called grouping mode(i.e. recovering the peaks in a mass spectrum from the points in a KMD plot using an appropriate software such as msRepeatFinder, JEOL).

\subsection{Versatility of a HRKMD analysis: point alignments vs. separation}

In spite of an indubitable improvement of the visualization, the series (1) and (3c) are found at almost the same KMD value owing to the aliasing of the KMD plot ${ }^{26-28)}$ and are differentiated mainly by the difference of their NKM (Fig. 2C). Such overlapping is more pronounced for the KMD plots computed with EO/45 from the SEC-MALDI mass spectral data (Fig. 3, top) as the mass range is restricted by the SEC fractionation(Fig. S2, Supporting Information). The choice of $X=45$ as a divisor for the computation of a high-resolution plot relies on a mathematical rationale proposed elsewhere ${ }^{28)}$. A divisor X close to the nominal mass of the repeat unit (round (repeat unit), e.g. 44 for EO) offers indeed a controlled gain of resolution through a limited expansion of the KMD dimension. It has

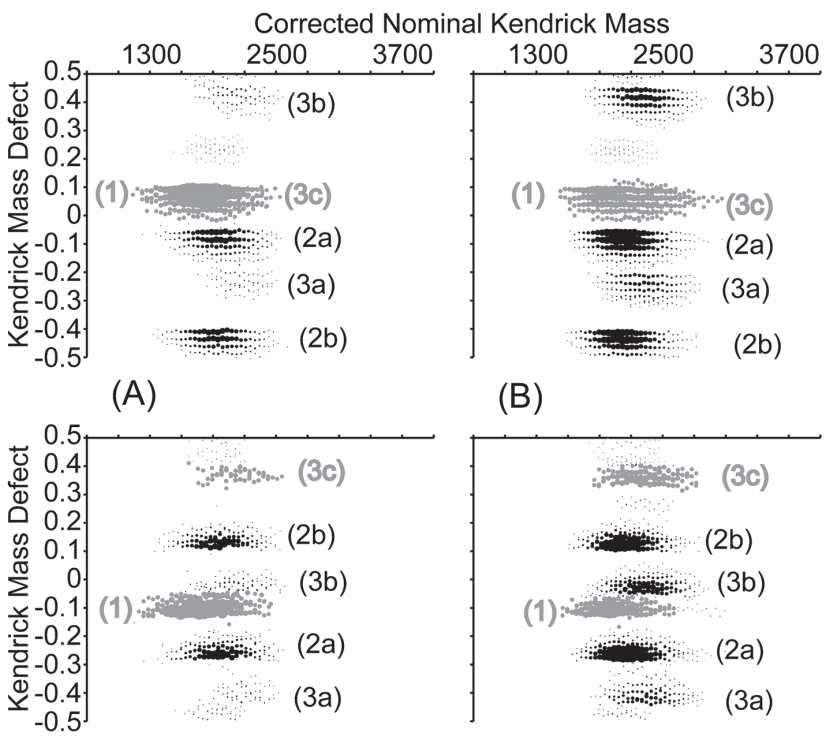

Fig. 3 HRKMD plots from the SEC-fractionated PEO "monostearate". (A) Fraction 2 (mass range: 13002400). (B) Fraction 3 (mass range: 1600-2700). Top: $\mathrm{EO} / 45$ as the base unit. Bottom: $\mathrm{EO} / 87$ as the base unit. (1: $(\mathrm{H}, \mathrm{OH}), 2 \mathrm{a}:(\mathrm{H}$, stearate $), 2 \mathrm{~b}:(\mathrm{H}$, palmitate), 3a (stearate, stearate), 3b (palmitate, stearate), 3c (palmitate, palmitate)-ended PEO), Overlapped series are highlighted in grey. 
been also stated that a divisor set at twice the nominal mass of the repeat unit (2*round (repeat unit), e.g. 88 for $\mathrm{EO})$ leads to poorer point alignments owing to the accumulation of errors but increases the quality of separation. The KMD plots computed with EO/87 as the base units are depicted in Fig. 3(bottom). The point alignments are clearly degraded (fuzzier appearance and absence of clear isotopic resolution) but the separation between the ion series (1) and $(3 \mathrm{c})$ is improved with no overlapping in spite of a similar mass range. The six point clouds are now isolated at full scale in terms of NKM and KMD. It highlights the versatility of this newly introduced HRKMD analysis computed with a fractional base unit as the divisor could be adjusted depending on the requirements such as a perfect point alignment, the isotopic resolution or the discrimination of components in blend.

\subsection{Representativeness of a direct MALDI-HRMS / HRK- MD analysis}

Several attempts of sample fractionation relying on a) the size of the analytes in solution (namely LC in SEC mode ${ }^{44)}$ ), b) the end-groups of the analytes (namely LC in adsorption mode LAC or at the critical condition $\mathrm{LCCC}^{37-39)}$ ) and c) the shape of the analytes in the gas phase $\left(\mathrm{IM}^{40)}\right)$ as well as alternative ionization mode (namely ESI) have been conducted to check at the representativeness of the direct MALDI-MS results reported above and interpreted with a HRKMD analysis. In spite of a size-based separation process with no chemical interaction with the stationary phase, the SEC-MALDI-MS analysis of PEO "monostearate" (Fig. S2) becomes an end-group profiling technique with the sequential detection of the distributions (1), (2) and (3) in the reverse order of elution (from low to high mass range). Interestingly, the same distributions (mainly mono- and di-esterified series (2) and (3)) are detected with two maxima at $\sim 2500 \mathrm{Da}$ and $\sim 3500 \mathrm{Da}$ in the first fraction of high mass range (Fig. S2E). With the detection of the distribution(1) at $\sim 2800 \mathrm{Da}$, such a bimodal shape might arise from a contamination by another grade of PEO "monostearate" with longer PEO chains (e.g. 65 or 70 EO) during the elaboration of the chemical at the factory. With no consideration for the variation of intensities, all the species found sequentially in a SEC-MALDI-MS analysis have been detected in the sufficiently robust direct MALDIMS analysis.

Similarly, the ESI mass spectrum of PEO "monostearate" is depicted in Fig. S3 and displays the same distributions ( (1) to (3) at +2 and +3 charge states. A strong suppression of signal is nevertheless observed with the dominion of polar species (mainly $(\mathrm{H}, \mathrm{OH})-\mathrm{PEO},(1))$ detrimentally to the di-esterified chains (3a)-(3c). A MALDI-MS analysis is more robust towards such polar/apolar discrimination without the absolute need for a preliminary LC fractionation. A rapid screening of chromatography conditions led to the LCCC-MS profile at the critical adsorption point of $\mathrm{EO}^{45)}$ depicted in Fig. S4. The polar distribution(1) and the mono-esterified ( $\mathrm{H}$, myristate), (2b) and (2a) are detected with satisfactory intensities in that order of elution on a reversed-phase column followed by the most apolar di-esterified series (3c-a) with no other components, validating the composition proposed from the direct MALDIMS analysis. A last way to fractionate the sample has been conducted by IM-MS. All the six distributions (1), (2a, 2b) and (3a-c) are extracted from the full mobilogram depicted in Fig. S5 with a strong signal suppression effect(ESI ion source with no preliminary fractionation). A restricted mobilogram (Fig. S6) displays three sets of spots readily assigned to (1), (2a) and (3a) doubly charged species. If the mobility dimension offers an alternative way to highlight the occurrence of these compounds by deconvoluting the overlapped peaks (Fig. 2A), the resolution of the spiralTOF mass analyzer is high enough to separate these components by their $m / z$ values only without the absolute need for an orthogonal separation step. In other words and in spite of the inherent richness of the sample, a direct MALDI-MS analysis combined with a HR-KMD analysis is sufficiently free of bias to provide a complete overview of the PEO "monosterate" sample. The same analytical strategy has been consequently employed to characterize the more complex ethoxylated hydrogenated castor oil (EHCO) sample.

\subsection{MALDI-MS / HRKMD analyses of EHCO}

The MALDI mass spectrum of EHCO with a degree of ethoxylation of ten(i.e. $10 \mathrm{EO}$ units) as mentioned by the supplier is depicted in Fig. 4A. A set of five main groups of peaks themselves composed of approximatively 10-14 peaks spaced by $44.0262 \mathrm{Da}$ (EO unit)are readily seen. Relying on the mass accuracy of the spiralTOF analyzer, two peaks from two groups are spaced by a generic $x^{*} 44.0262+y * 282.2559$ Da mass difference, i.e. $x$ EO units and y dehydrated hydrogenated ricinoleic acid moieties $\left(\mathrm{C}_{18} \mathrm{H}_{34} \mathrm{O}_{2},-\mathrm{H}_{2} \mathrm{O}\right.$ as compared to the hydrogenated ricinoleic acid $\mathrm{C}_{18} \mathrm{H}_{36} \mathrm{O}_{3}$ ). The latter neutral corresponds to a hydrogenated ricinoleic acid moiety involved in an ester bond such as the end-group of a PEO chain. Considering this varying number of $\mathrm{EO}$ and hydrogenated ricinoleate, a simplified notation $(\mathrm{x}, \mathrm{y})$ is proposed and exemplified in Fig. 4A with peaks carrying $10 \mathrm{EO}$ units $(\mathrm{x}=10)$ and a varying number of hydrogenated ricinoleate moieties y. For example, the sodiated $(\mathrm{x}=10, \mathrm{y}=0)$ species at $m / z 555.3$ is composed of a glycerol initiating end group, $10 \mathrm{EO}$ units and $\mathrm{a}-\mathrm{OH}$ terminating end-group while $\mathrm{a}(\mathrm{x}=10, \mathrm{y}=2)$ sodium adduct at $\mathrm{m} / \mathrm{z} 1119.8$ contains two hydrogenated ricinoleate in its backbone. The main peaks detected in the mass spectrum of EHCO can thus been described as cooligomers of $\mathrm{EO}$ and hydrogenated ricinoleate carrying (glycerol, OH) terminations and the corresponding diblock 


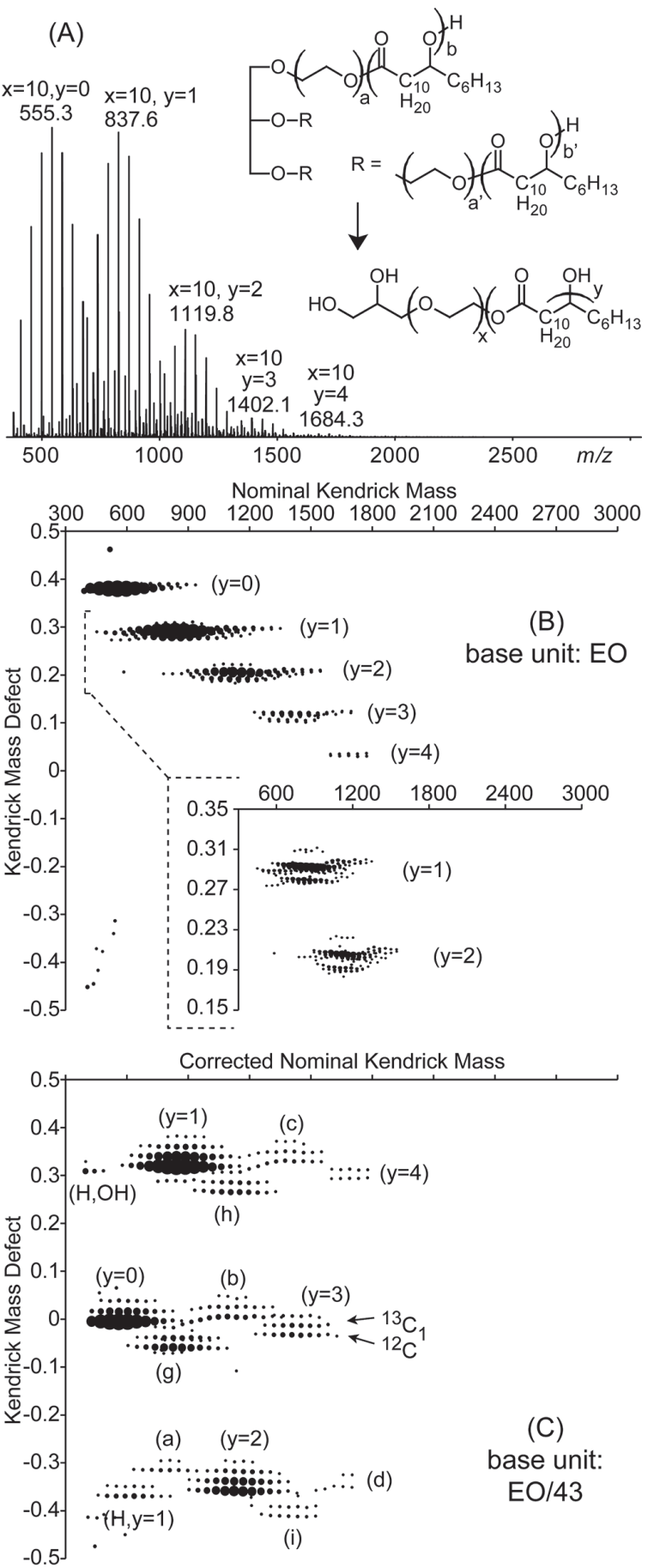

Fig. 4 (A) MALDI mass spectrum of EHCO. Insets: triglyceride and di-block simplified structure of the main components (x: polymerization of $\mathrm{EO}, \mathrm{y}$ : polymerization of hydrogenated ricinoleate). (B) Regular KMD plot using EO as the base unit. (C) HRKMD plot using EO/43 as the base unit. ( $\mathrm{y}=$ $0-4)$ : (glycerol, OH) -PEO + 0-4*hydrogenated ricinoleate. $(\mathrm{a}-\mathrm{d}):(\mathrm{x}, \mathrm{y}=0-3)+$ glycerol. $(\mathrm{g}-\mathrm{i}):(\mathrm{x}$, $\mathrm{y}=0-2)+$ stearate. $(\mathrm{H}, \mathrm{OH}):(\mathrm{H}, \mathrm{O})-\mathrm{PEO} .(\mathrm{H}, \mathrm{y}=$ 1): ( $\mathrm{H}$, hydrogenated ricinoleate) $-\mathrm{PEO}$. copolymeric structure is depicted in inset in Fig. 4A (simplified structure derived from the triglyceride structure proposed in the literature). Accurate mass measurements, assignments and KMDs are listed in Table S2 in the Supporting Information.

As for the PEO "monostearate" sample, a description of the raw mass spectral data is tedious and could be greatly facilitated using a KMD analysis. The regular KMD plot computed with EO as the base unit is depicted in Fig. 4B. The five main groups of points detected in the mass spectrum are now seen as five horizontal lines discriminating the species based on the number of fatty acid moieties $(y=$ 0-4). A magnification of two point clouds reveals a more complex pattern with several sets of horizontal lines arising from several by-products. Owing to the need for zooming, points are barely align along the horizontal axis (imperfect calibration), several groups of points are not properly separated (low variation of KMD) and the isotopic resolution is not reached.

Computing a HRKMD plot with EO/43 as the base unit dramatically improves the resolution and corrects all the bias mentioned above (Fig. 4C). In addition to the (x, y) ion series with $y=0-4$, several point clouds are now properly highlighted and assigned to polyglycerol polyricinoleate side $\operatorname{products}^{23)}$ (formally (x, y=0-3) + glycerol, noted (a-d) ) and stearate-containing byproducts (formally $(\mathrm{x}, \mathrm{y}=$ $0-2)+$ stearate, noted $(\mathrm{g}-\mathrm{i}))$. As for PEO "monostearate", using a fractional base unit with a divisor set close to the nominal mass of the repeat unit expands the KMD dimension in a controlled manner and ion series are satisfactorily separated $((\mathrm{y}=1)$ vs. $(\mathrm{y}=0)+$ stearate: $\Delta \mathrm{KMD}=0.0146$ with $\mathrm{EO}$ and $\Delta \mathrm{KMD}=0.3779$ with $\mathrm{EO} / 43$, gain of resolution $\mathrm{x} 26)$. Two additional ion series are also isolated in the low NKM range and assigned to a $(\mathrm{H}, \mathrm{OH})-\mathrm{PEO}$ and a glycerolfree $(\mathrm{H}$, hydrogenated ricinoleate) $-\mathrm{PEO}(\operatorname{noted}(\mathrm{H}, \mathrm{y}=1)$ in Fig. $4 \mathrm{C})$. It is noteworthy that $(\mathrm{H}, \mathrm{OH})-\mathrm{PEO}$ and $(\mathrm{x}, \mathrm{y}=0)$ ion series have nearly the same mass defect (at $\sim 0.0245$ and $\sim 0.0318, \Delta \mathrm{KMD} \sim 0.0073$ ) and cannot be isolated in a regular KMD plot regardless of the magnification, requiring a HRKMD analysis to be separated $(\triangle \mathrm{KMD} \sim 0.3111)$.

The generic structure depicted in Fig. 4A is supported by the ESI tandem mass spectra recorded from the $(\mathrm{x}=10$, y) congeners with $y=1-5$ (Fig. S7). In particular, several hydrogenated ricinoleate moieties are highly suspected to be linked via covalent bonds in a block shape rather than distributed. Such description surprisingly departs from the triglyceride structure expected for the castor oil (up to three esters of fatty acids ${ }^{18-24}$ but is in accordance with previous results indicating the presence of supernumerary fatty acid moieties ${ }^{19}$ and structural defects such as glycerol-free chains and ricinoleate-ricinoleate bond ${ }^{17)}$. Similarly, the PEO chain is not distributed between glycerol and the fatty acid moieties (neutrals expelled in tandem mass spectra would contain a varying number of EO units) but 
consists of a block schematized by a glycerol-PEO-(fatty acid) ${ }_{y}-\mathrm{OH}$ generic structure. Echoing the strategy reported for PEO "monostearate" and since a direct MALDI-MS analysis of castor oil sample might be oversimplified in terms of detected species, a set of orthogonal fractionation techniques has been used to check at the representativeness of the MALDI-HRMS / HRKMD analysis. A fractionation of species based on their content in hydrogenated ricinoleate is achieved by SEC-MALDI-MS with the detection of $(x, y=0),(x, y=1),(x, y=2-4),(x, y=3-7),(x, y=$ $6-11)$ and $(x, y=9-14)$ in the reverse order of elution (Fig. S8). The ESI mass spectrum of EHCO (Fig. S9) displays the distributions $(\mathrm{x}, \mathrm{y}=0-2)$ highlighting once more a strong suppression of signal detrimentally to the hydrogenated ricinoleate-rich species $(y>2)$. The $(x, y=4-0)$ are detected in that order of elution in the LC-MS profile (LAC mode on a normal phase column, Fig. S10). The (H, OH) -PEO distribution detected as a major component in the late elution times between $(\mathrm{x}, \mathrm{y}=1)$ and $(\mathrm{x}, \mathrm{y}=0)$ was nevertheless already highlighted in the MALDI-HRMS/HRKMD analysis thanks to the gain of resolution offered by the fractional base units. The last fractionation of the sample by ESI-IM is also subjected to a strong suppression of the signal for the most apolar species with the detection of $(\mathrm{x}$, $\mathrm{y}=0-2)$ as the main series in addition to the $(\mathrm{H}, \mathrm{OH})-\mathrm{PEO}$ distribution in a full mobilogram(Fig. S11). A restricted mobilogram centered around $(\mathrm{x}=10, \mathrm{y}=2)$ at $\mathrm{m} / z 1119$ (Fig. S12) displays three spots assigned to three isomeric forms (same $\mathrm{m} / z$, different drift time $=$ different shape in the gas phase). Three MS/MS fingerprints in the IM tandem mass spectra confirm the presence of isomers behind a single $m / z$ (e.g. position of the two hydrogenated ricinoleate as end-groups or within a glycerol-PEO backbone). If each of these techniques provide complimentary information about EHCO, all the species above-mentioned have been observed in the direct MALDI HRMS analysis combined with a HRKMD analysis, exemplifying once more the capability of such analytical strategy for the characterization of complex samples.

As for PEO "monostearate", using a divisor set at 87 in lieu of 43 further improves the resolution of the KMD analysis (Fig. 5). Eighteen ion series are highlighted in the HRKMD plots computed with EO/43 from two SEC-MALDI mass spectra (Fig. S8) belonging to the main three classes $(\mathrm{x}, \mathrm{y}=1-7),(\mathrm{x}, \mathrm{y}=0-5)+$ glycerol noted $(\mathrm{a}-\mathrm{f})$ and $(\mathrm{x}, \mathrm{y}=$ 0-6) + stearate noted (g-1) (Fig. 5, top). Computed with $\mathrm{EO} / 87$, the separation of these eighteen groups is improved and also allows the assignment of four additional ion series described as a generic $(x, y=2-5)+$ glycerol + stearate $(\operatorname{noted}(\mathrm{m}-\mathrm{p}))$. Following a thorough analysis of the two pristine non-ionic surfactants, such an unrivaled tool for the data mining of rich mass spectral data could be safely used for the characterization of aged samples whose complexity might be increased by the degradation step.

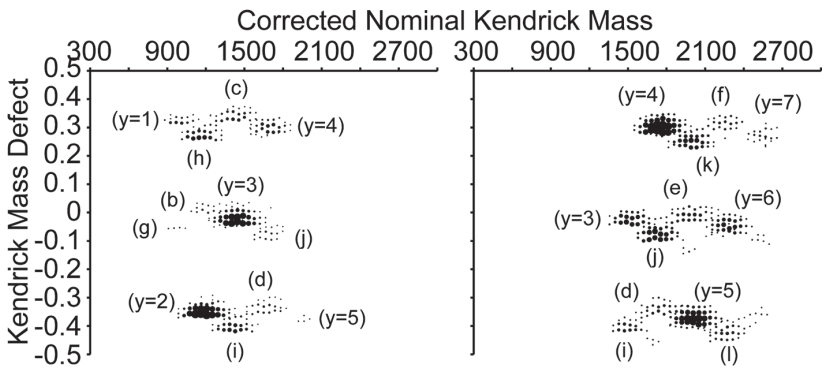

(A)

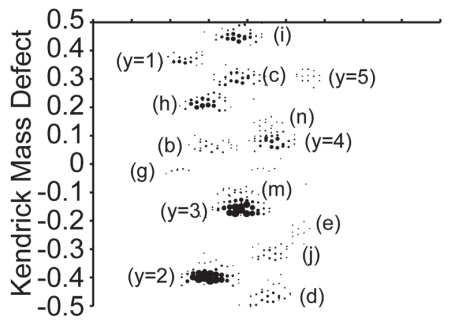

(B)

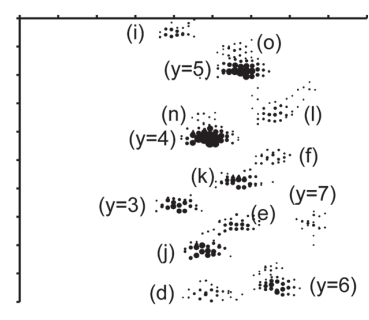

Fig. 5 HRKMD plots from the SEC-fractionated EHCO. (A) Fraction 3 (mass range: 900-1900). (B) Fraction 4 (mass range: 1300-2700). Top: EO/43 as the base unit. Bottom: EO/87 as the base unit. (a-f) $=(\mathrm{x}, \mathrm{y}=0-5)+$ glycerol; $(\mathrm{g}-\mathrm{l})=(\mathrm{x}, \mathrm{y}=0-6)+$ stearate; $(\mathrm{m}-\mathrm{p}):(\mathrm{x}, \mathrm{y}=2-5)+$ glycerol + stearate.

\subsection{HRKMD analysis of degraded surfactants}

The MALDI mass spectrum of a PEO "monostearate" sample let to react for 15 days in a phosphate buffer is depicted in Fig. 6A. At first sight, the (H, OH)-PEO series (1) is noteworthy of higher intensity and several peaks are detected in the low mass range but the whole fingerprint does not strikingly depart from its pristine counterpart (Fig. 2A). Instead of a tedious description of the mass spectrum with the need for multiple magnifications but relying on the enhanced visualization offered by a KMD analysis, the HRKMD plot of the aged sample computed with $\mathrm{EO} / 45$ is proposed in Fig. $6 \mathrm{~B}$ and clearly differs from the KMD plot of the unaged sample (Fig. 2C).

Three main features are readily observed and attributed to two ageing routes. First and as mentioned, the intensity of the $(\mathrm{H}, \mathrm{OH})-\mathrm{PEO}(1)$ is noteworthy increased and might be accounted for by the expected hydrolytic degradation of the series (2) and (3) via the cleavage of the ester bond ${ }^{46)}$ (Fig. 6C). Second, several new distributions are detected through the display of new point clouds in the KMD plot. Third, several distributions clearly expand towards the lower mass range. From a mechanistic point of view, the last two points mean new end-groups have been formed (new groups of points) upon a chain scission mechanism (expansion towards low NKM) such as an oxidative degradation $^{47,48)}$ (e.g. autooxidation of ethers, Fig. 6C) in addition to the expected sole hydrolysis of the ester groups. Points have been grouped using a light grey line for the sake of an easy description but the separation of points is indisput- 

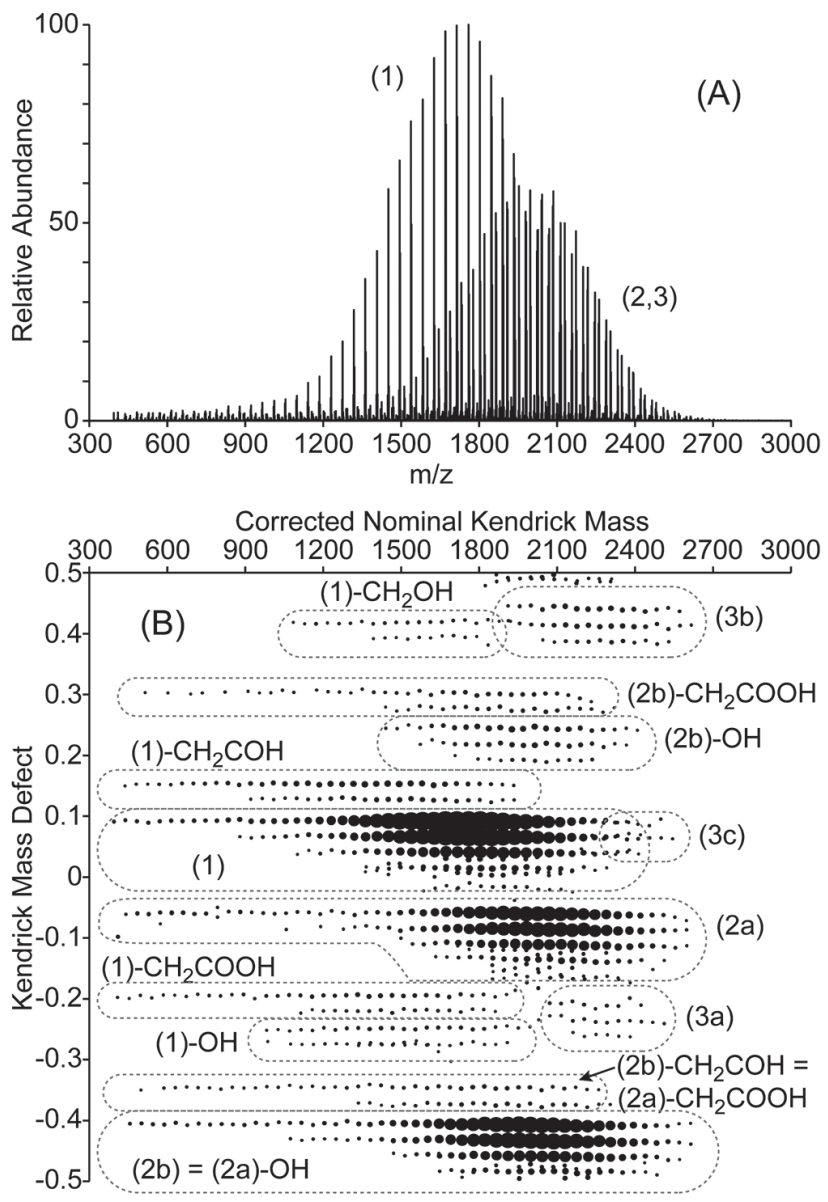

Fatty ester $(\text { EO })_{x}$ Fatty ester $\stackrel{\text { Hydrolysis }}{\longrightarrow}$ Fatty ester $(\mathrm{EO})_{x} \mathrm{OH}$

(3)

(2)

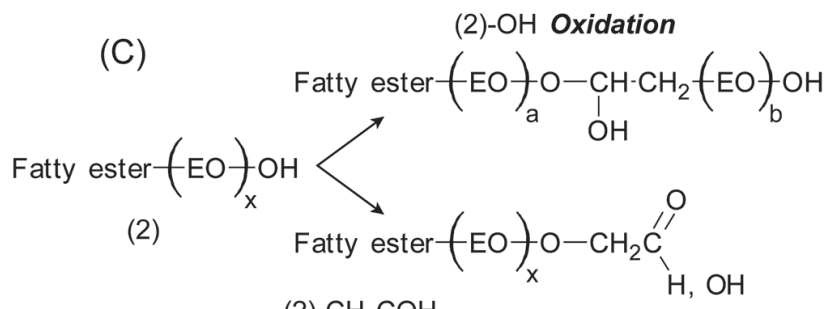

(2) $-\mathrm{CH}_{2} \mathrm{COH}$

(2) $-\mathrm{CH}_{2} \mathrm{COOH}$ Oxidative chain scission

Fig. 6 (A) MALDI mass spectrum of a PEO "monostearate" after 15 days of immersion in a phosphate buffer at $40^{\circ} \mathrm{C}$. (B) HRKMD plot using $\mathrm{EO} / 45$ as the base unit (1: (H, OH-PEO, 2a: $(\mathrm{H}$, stearate), 2b: (H, palmitate), $3 \mathrm{a}$ (stearate, stearate), 3b (palmitate, stearate), 3c (palmitate, palmitate)-ended $\mathrm{PEO}$ ). (x)- $\mathrm{CH}_{2} \mathrm{COH}$ and (x) - $\mathrm{CH} 2 \mathrm{COOH}$ refer to an aldehyde and carboxylic ended PEO series formed upon its oxidative chain cleavage, (x)-OH refers to an oxidized chain carrying the original terminations. (C) Main degradation pathways. ably improved as compared to a regular KMD plot computed with EO(Fig. S13, Supporting Information). In addition to the pristine series (1-3), distributions assigned to an aldehyde-ended and a carboxylic ended-PEO and carrying either the unreacted $-\mathrm{OH}$ end-group (noted (1) $-\mathrm{CH}_{2} \mathrm{COH}$ and (1) $-\mathrm{CH}_{2} \mathrm{COOH}$ ) or one untouched fatty acid (and noted (2a) - or (2b) - $\mathrm{CH}_{2} \mathrm{COH}$ and (2a) - or $\left.(2 \mathrm{~b})-\mathrm{CH}_{2} \mathrm{COOH}\right)$ are readily isolated. Two last series assigned to an oxidized $\mathrm{PEO}$ chain carrying a supernumerary $=\mathrm{O}$ group are observed from (1) and (2b) and noted (1)-OH and (2b)-OH confirming an oxidative route occurred during the degradation test. Relying on the literature, end-groups of the PEO distributions are foreseeable and the associated KMD values are simulated accordingly. Point lines in the KMD plots are thus readily assigned with no need for a manual and time-consuming peak-picking in the raw mass spectrum. Accurate mass measurements, assignments and KMDs are listed in Table S3. In spite of the richness of the degraded sample, the KMD analysis in its high-resolution form thus provides valuable information, an indubitable enhanced visualization of data and a great saving of time by simply comparing the KMD plots (Fig. 2C and Fig. 6B) .

Interestingly, the pristine PEO "monostearate" sample has been proposed as a model for the implementation of the analytical strategy relying on its relative simplicity compared to the complexity of EHCO. However, the aged PEO "monostearate" has been found of greater complexity with the occurrence of two competitive degradation route while the fingerprint of a degraded EHCO sample(Fig. 7) appears dramatically simpler. The MALDI mass spectrum of a 15-day aged EHCO in a phosphate buffer at $40^{\circ} \mathrm{C}$ is depicted in Fig. 7A and displays a reduced number of distributions as compared to its pristine counterpart (Fig. 4A). This impression is confirmed by the HRKMD plot computed with EO/43 which displays only the low molecular weight distributions described from Fig. 4B carrying only one, two or no hydrogenated ricinoleate moieties $(\mathrm{x}, \mathrm{y}=0-3)$ (Fig. 7B). Such fingerprint is undoubtedly arising from a hydrolytic degradation of the surfactant through the cleavage of the ester bond (Fig. 7C ${ }^{46)}$. Accurate mass measurements, assignments and KMDs are listed in Table S4.

As a last digression, the fingerprint of the degraded sample also tends to validate the generic structure proposed in the previous section and consisting in a PEO block capped with a glycerol moiety as the initiating endgroup and a varying number of fatty acids linked to each other in a second block (inset in Fig. 4A). It then turns the degradation step into another analytical tool to gain structural insights into the intact sample as proposed in previous articles for the unravelling of complex polymeric networks $^{49,50)}$. 


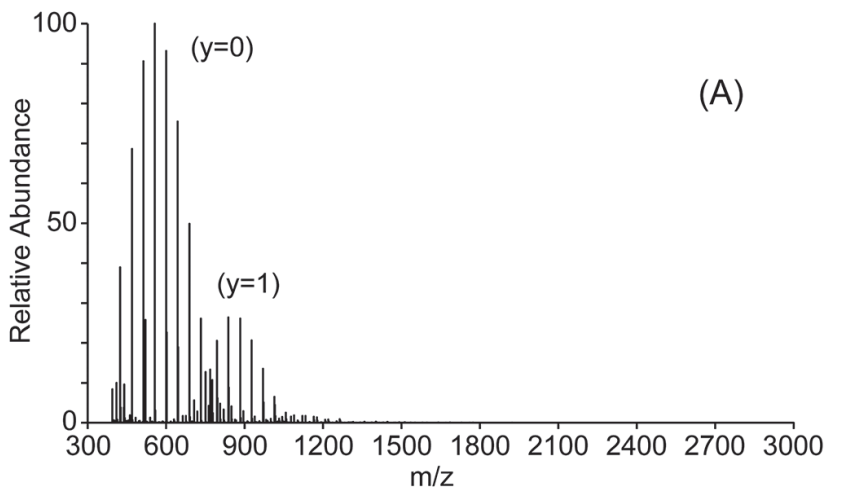

Corrected Nominal Kendrick Mass

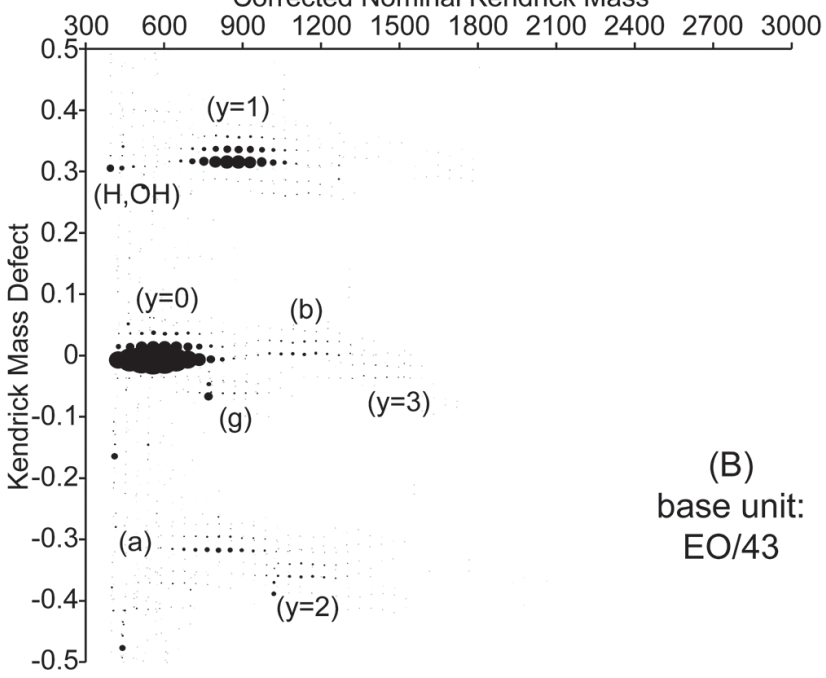

(C) $(x, y)$ $(\mathrm{x}, \mathrm{y}=0)$<smiles>[Y]C(C)(O)CCOC(C)(C)C(O)CO</smiles>

Fig. 7 (A) MALDI mass spectrum of a EHCO sample after 15 days of immersion in a phosphate buffer at $40^{\circ} \mathrm{C}$. (B) HRKMD plot using EO/43 as the base unit. $(y=0-3)$ : (glycerol, OH)-PEO + $\mathrm{y}^{*}$ hydrogenated ricinoleate; $(\mathrm{a}, \mathrm{b}):(\mathrm{x}, \mathrm{y}=0,1)+$ glycerol. $(\mathrm{g}):(\mathrm{x}, \mathrm{y}=0)+$ stearate. $(\mathrm{H}, \mathrm{OH}):(\mathrm{H}, \mathrm{O})$ -PEO. (C) Main degradation pathway.

\section{CONCLUSION}

A demonstration of the capabilities of a combined MALDI-HRMS / HRKMD analysis is reported with a detailed characterization of pristine and aged non-ionic PEO-based surfactants, sufficiently free of bias to provide a complete overview of the samples as compared to orthogonal analytical techniques (alternative ionization, fractionations). In particular, a HRKMD analysis strikingly improved the visualization and interpretation of data and avoided a tedious and time-consuming peak assignment of complex mass spectra. A surprising reversal of the situation has also been found in terms of complexity of the samples. The relatively simple PEO "monostearate" has been used as a reference sample for the analysis of a more complex castor oil derivative. Upon ageing, the castor oil sample has been found to undergo a hydrolytic degradation only with a simple MS and KMD fingerprint while additional oxidative chain scission routes were identified for PEO "monostearate" from its complex mass spectra and KMD plots. This first application of HRKMD on actual samples is presaging a bright future for this data mining tool. It is expected to become of routine usage for an enhanced visualization and easy interpretation of mass spectral data thanks to its recent incorporation in commercially available softwares. More complex surfactants (other bio-based reactants, ionic surfactants...), full formulations of final products (cosmetics, detergents...) or samples submitted to other ageing routes (chemolysis, thermo-oxidation, UV...) would be advantageously investigated using the MALDI-HRMS / HRKMD combination to further exemplify the robustness of the method.

\section{Acknowledgements}

T. Fouquet and H. Sato acknowledge the ongoing financial support by the Japan Society for the Promotion of Science (JSPS) under the postdoctoral fellowship for overseas researchers program (FY2015) and a Grant-in-Aid “JSPS KAKENHI” (Grant number: JP 15F15344).

\section{Supporting information}

This material is available free of charge via the Internet at http://dx.doi.org/jos.66.10.5650/jos.ess.17096

\section{REFERENCES}

1) Salager, J.-L. Surfactant's types and uses. Firp Booket-E300-Attaching Aid in Surfactant Science and Engineering in English. University of Andes, Meridia, Venezuela (2002).

2) Ramanathan, M.; Shrestha, L.K.; Mori, T.; Ji, Q.; Hill, J.P.; Ariga, K. Amphiphile nanoarchitectonics: from basic physical chemistry to advanced applications. Phys. Chem. Chem. Phys. 15, 10580-10611 (2013).

3) Manaargadoo-Catina, M.; Ali-Cherifa, A.; Pougnasa, J.L.; Perrin, C. Hemolysis by surfactants - A review. Adv. Colloid Interface Sci. 228, 1-16(2016).

4) Lee, C.H.; Jung, H.; Jo, D.H.; Jeon, S.; Kim, S.H. Effect of Surfactant on $\mathrm{CO}_{2}$ adsorption of APS-grafted silica gel by one-pot process. Bull. Chem. Soc. Jpn. 89, 823-832 (2016). 
5) Bakshi, M.S. How surfactants control crystal growth of nanomaterials. Cryst. Growth Des. 16, 1104-1133 (2016).

6) Miyabe, K.; Suzuki, N. Kinetic study of solute permeation across surfactant micelle/bulk solvent interface by moment analysis - chromatographic capillary electrophoresis(MA-CCE). Bull. Chem. Soc. Jpn. 89, 1212-1218(2016).

7) Cullum, D.C. Introduction to Surfactant Analysis. Springer, Netherland (1994).

8) Stubiger, G. ; Pittenauer, E.; Allmaier, G. Characterisation of castor oil by on-line and off-line non-aqueous reverse-phase high performance liquid chromatography-mass spectrometry (APCI and UV/MALDI) . Phytochem. Anal. 14, 337-346 (2003).

9) Bataglion, G.A.; da Silva, F.M.A.; Santos, J.M.; dos Santos, F.N.; Barcia, M.T.; de Lourenço, C.C.; Salvador, M.J.; Godoy, H.T.; Eberlin, M.N.; Koolen, H.H.F. Comprehensive characterization of lipids from Amazonian vegetable oils by mass spectrometry techniques. Food Res. Int. 64, 472-481 (2014).

10) Tran, N.B.; Vialle, J.; Pham, Q.T. Castor oil-based polyurethanes: 1. Structural characterization of castor oil nature of intact glycerides and distribution of hydroxyl groups. Polymer 38, 2467-2473(1997).

11) Kaushik, A.; Singh, P. Synthesis and characterization of castor oil/trimethylol propane polyol as raw materials for polyurethanes using time-of-flight mass spectroscopy. Int. J. Polymer Anal. Charact. 10, 373-386 (2005).

12) Benes, H.; Slaba, J.; Walterova, Z.; Rais, D. Recycling of waste poly (ethylene terephthalate) with castor oil using microwave heating. Polym. Degrad. Stab. 98, 2232-2243 (2013).

13) Spontón, M.; Casis, N.; Mazo, P.; Raud, B.; Simonetta, A,; Ríos, L.; Estenoz, D. Biodegradation study by Pseudomonas sp. of flexible polyurethane foams derived from castor oil. Int. Biodeterior. Biodegrad. 85, 85-94 (2013).

14) Halek, F.; Delavari, A.; Kavousi-rahim, A. Production of biodiesel as a renewable energy source from castor oil. Clean Techn. Environ. Policy 15, 1063-1068 (2013).

15) Kunduru, K.R.; Basu, A.; Zada, M.H.; Domb, A.J. Castor oil-based biodegradable polyesters. Biomacromolecules 16, 2572-2587 (2015).

16) Zhang, Q.; Sun, Y.; Zhi, L.; Zhang, Y.; Di Serio, M. Properties of ethoxylated castor oil acid methyl esters prepared by ethoxylation over an alkaline catalyst. $J$. Surfact. Deterg. 18, 365-370 (2015).

17) Nasioudis, A.; van Velde, J.W.; Heeren, R.M.A.; van den Brink, O.F. Detailed molecular characterization of castor oil ethoxylates by liquid chromatography multistage mass spectrometry. J. Chromatogr. A 1218,
7166-7172(2011).

18) Ramamurthi, S.; Manohar, V.; Mani, V.V.S. Characterization of fatty acid isomers in dehydrated castor oil by gas chromatography and gas chromatography-mass spectrometry techniques. J. Am. Oil Chem. Soc. 75, 1297-1303 (1998).

19) Lin, J.-T.; Arcinas, A.; Harden, L.R.; Fagerquist, C.K. Identification of (12-Ricinoleoylricinoleoyl) diricinoleoylglycerol, an acylglycerol containing four acyl chains, in castor (Ricinus communis L.) oil by LCESI-MS. J. Agric. Food Chem. 54, 3498-3504 (2006).

20) Lin, J.-T.; Arcinas, A. Regiospecific analysis of diricinoleoylacylglycerols in castor (Ricinus communis L.) oil by electrospray ionization-mass spectrometry. $J$. Agric. Food Chem. 55, 2209-2216 (2007).

21） Lin, J.-T. Ratios of regioisomers of triacylglycerols containing dihydroxy fatty acids in castor oil by mass spectrometry. J. Am. Oil Chem. Soc. 86, 1031-1035 (2009).

22) Lin, J.-T.; Chen, G.Q. Identification of diacylglycerol and triacylglycerol containing 11,12,13-trihydroxy-9,14-octadecadienoic acid in castor oil. N. Biotechnol. 28, 203-208(2011).

23) Lin, J.-T.; Chen, G.Q. Identification of minor acylglycerols less polar than triricinolein in castor oil by mass spectrometry. J. Am. Oil Chem. Soc. 89, 1773-1784 (2012).

24) Silva, R.V.S.; Casilli, A.; Sampaio, A.L.; Avila, B.M.F.; Veloso, M.C.C.; Azevedo, D.A.; Romeiro, G.A. The analytical characterization of castor seed cake pyrolysis bio-oils by using comprehensive GC coupled to time of flight mass spectrometry. J. Anal. Appl. Pyrolysis 106, 152-159 (2014).

25) Pasch, H.; Schrepp, W. MALDI-TOF mass spectrometry of synthetic polymers. Springer-Verlag (2003).

26) Fouquet, T.; Sato, H. Improving the resolution of Kendrick mass defect analysis for polymer ions with fractional base units. Mass Spectrom. (Tokyo)6, A0055 (2017).

27) T. Fouquet, H. Sato. Extension of the Kendrick Mass Defect Analysis of Homopolymers to Low Resolution and High Mass Range Mass Spectra Using Fractional Base Units. Anal. Chem. 89, 2682-2686 (2017).

28) Fouquet, T.; Sato, H. How to choose the best fractional base unit for a high resolution Kendrick mass defect analysis of polymer ions. Rapid Commun. Mass Spectrom. 31, 1067-1072(2017).

29) Sato, H.; Nakamura, S.; Teramoto, K.; Sato, T. Structural characterization of polymers by MALDI SpiralTOF mass spectrometry combined with Kendrick mass defect analysis. J. Am. Soc. Mass Spectrom. 25, 13461355 (2014).

30) Fouquet, T.; Aizawa, H.; Sato, H. Taking MALDI SpiralTOF high-resolution mass spectrometry and mass de- 
fect analysis to the next level with ethylene vinyl acetate vinyl alcohol terpolymers. Rapid Commun. Mass Spectrom. 30, 1818-1822 (2016).

31) Fouquet, T.; Nakamura, S.; Sato, H. MALDI SpiralTOF high-resolution mass spectrometry and Kendrick mass defect analysis applied to the characterization of poly (ethylene-co-vinyl acetate) copolymers. Rapid Commun. Mass Spectrom. 30, 973-981(2016).

32) Fouquet, T.; Torimura, M.; Sato, H. Multi-stage mass spectrometry of poly (vinyl pyrrolidone) and its vinyl succinimide copolymer formed upon exposure to sodium hypochlorite. Mass Spectrom. (Tokyo) 5, A0050 (2016).

33) Xing, Z. W.; Ke, H.T.; Wang, J.R.; Zhao, B.; Yue, X.L.; Dai, Z.F.; Liu, J.B. Novel ultrasound contrast agent based on microbubbles generated from surfactant mixtures of Span 60 and polyoxyethylene 40 stearate. Acta Biomater. 6, 3542-3549 (2010).

34) Cassano, R.; Ferrarelli, T.; Mauro, M.V.; Cavalcanti, P.; Picci, N.; Trombino, S. Preparation, characterization and in vitro activities evaluation of solid lipid nanoparticles based on PEG-40 stearate for antifungal drugs vaginal delivery. Drug Deliv. 23, 1047-1056 (2016).

35) Yacob, A.R.; Bello, A.M.; Kabo, K.S. The effect of polyoxyethylene (40) stearate surfactant on novel synthesis of mesoporous gamma-alumina from Kano kaolin. Arab. J. Chem. 9, 297-304(2016).

36) Charles, L.; Altuntas, E. Liquid chromatography-electrospray ionization mass spectrometry of synthetic polymers. in Encyclopedia of Analytical Chemistry, Online c 2006-2015, John Wiley \& Sons, Ltd.

37) Nielen, M.W.F.; Buijtenhuijs, F.A. Polymer analysis by liquid chromatography/electrospray ionization time-offlight mass spectrometry. Anal. Chem. 71, 1809-1814 (1999).

38) Girod, M.; Phan, T.N.T.; Charles, L. On-line coupling of liquid chromatography at critical conditions with electrospray ionization tandem mass spectrometry for the characterization of a nitroxide-mediated poly (ethylene oxide)/polystyrene block copolymer. Rapid Commun. Mass Spectrom. 22, 3767-3775(2008).

39) Girod, M.; Beaudoin, E.; Charles, L. Effect of salt in the mobile phase on the critical conditions of poly (ethylene glycol) in liquid chromatography-mass spectrometry coupling. Anal. Methods 1, 128-131 (2009).

40) Wesdemiotis, C. Multidimensional mass spectrometry of synthetic polymers and advanced materials. Angew.
Chem. Int. Ed. 56, 1452-1464(2017).

41) Satoh, T.; Tsuno, H.; Iwanaga, M.; Kamei, Y. The design and characteristics features of a new time-of-flight mass spectrometer with a spiral ion trajectory. J. Am. Soc. Mass Spectrom. 16, 1969-1975(2005).

42) Strohalm, M.; Kavan, D.; Novák, P.; Volný, M.; Havlícek, V. mMass 3: a cross-platform software environment for precise analysis of mass spectrometric data. Anal. Chem. 82, 4648-4651 (2010).

43) Kendrick, E. A mass scale based on $\mathrm{CH}_{2}=14.0000$ for high resolution mass spectrometry of organic compounds. Anal. Chem. 35, 2146-2154(1963).

44) Gruendling, T.; Weidner, S.; Falkenhagen, J.; BarnerKowollik, C. Mass spectrometry in polymer chemistry: a state of the art up-date. Polym. Chem. 1, 599-617 (2010).

45) Malik, M.I.; Ahmed, H.; Trathnigg, B. Liquid chromatography under critical conditions: Practical applications in the analysis of amphiphilic polymers. Anal. Bioanal. Chem. 393, 1797-1804(2009).

46) Stjerndahl, M.; van Ginkel, C.G.; Holmberg, K. Hydrolysis and biodegradation studies of surface-active esters. J. Surfact. Deterg. 6, 319-324 (2003).

47) Sato, H.; Shibata, A.; Wang, Y.; Yoshikawa, H.; Tamura, $\mathrm{H}$. Characterization of biodegradation intermediates of non-ionic surfactants by matrix-assisted laser desorption/ionization-mass spectrometry 1 . Bacterial biodegradation of octylphenol polyethoxylate under aerobic conditions. Polym. Degrad. Stab. 74, 69-75 (2001).

48) Chen, L.; Yamane, S.; Sago, T.; Hagihara, H.; Kutsuna, S.; Uchimaru, T.; Suda, H.; Sato, H.; Mizukado, J. Experimental and modeling approaches for the formation of hydroperoxide during the auto-oxidation of polymers: Thermal-oxidative degradation of polyethylene oxide. Chem. Phys. Lett. 657, 83-89(2016).

49) Fouquet, T.; Bour, J.; Toniazzo, V.; Ruch, D.; Charles, L. Characterization of ethanolysis products of poly (dimethylsiloxane) species by electrospray ionization tandem mass spectrometry. Rapid Commun. Mass Spectrom. 26, 2057-2067 (2012).

50) Fouquet, T.; Petersen, J.; Ziarelli, F.; Bour, J.; Toniazzo, V.; Ruch, D.; Charles, L. Insights in the architecture of silicon-based plasma polymers using partial network ethanolysis combined with electrospray tandem mass spectrometry. Plasma Process. Polym. 10, 271-284 (2013). 